\title{
A NOTE ON PARABOLIC POWER CONCAVITY
}

\author{
Kazuhiro Ishige and Paolo Salani
}

\begin{abstract}
We investigate parabolic power concavity properties of the solutions of the heat equation in $\Omega \times[0, T)$, where $\Omega=\mathbf{R}^{n}$ or $\Omega$ is a bounded convex domain in $\mathbf{R}^{n}$.
\end{abstract}

\section{Introduction}

Consider the Cauchy-Dirichlet problem for the heat equation

$$
\begin{cases}\partial_{t} u=\Delta u & \text { in } \Omega \times(0, \infty), \\ u(x, t)=0 & \text { on } \partial \Omega \times(0, \infty) \text { if } \partial \Omega \neq \emptyset, \\ u(x, 0)=\varphi(x) \geq 0 & \text { in } \Omega,\end{cases}
$$

where $\partial t=\partial / \partial t, \Omega=\mathbf{R}^{n}$ or $\Omega$ is a bounded convex domain in $\mathbf{R}^{n}(n \geq 1)$ and $\varphi \in C(\Omega) \cap L^{\infty}(\Omega)$. This paper is concerned with concavity properties involving the space and the time variables jointly for the solution of (1.1).

Questions about geometric properties of solutions are quite natural in the theory of elliptic and parabolic equations. In this regard, convexity and convexity-like properties have been investigated extensively, especially in the field of elliptic equations. See [21] for a good introduction to this subject; see also $[2,3,20,25,29]$ as some of the most recent results, to our knowledge, for elliptic equations. A typical question is the following: if $u>0$ solves an elliptic Dirichlet problem in a bounded convex domain $\Omega$ with constant vanishing boundary data, does $u$ have to be concave? As it is easily seen, the answer to this question is in general negative: it is possible to construct examples in which concavity goes completely lost (see for instance [12]) and one can hardly expect a genuinely concave solution even to a simple Dirichlet problem like the following torsion problem

$$
\begin{cases}\Delta u=-1 & \text { in } \Omega \\ u=0 & \text { on } \partial \Omega\end{cases}
$$

1991 Mathematics Subject Classification. 35K05, 52A01, 35B05, 35B30.

Key words and phrases. power concavity, parabolic concavity, heat equation. Received April 1, 2014; revised May 7, 2014. 
where indeed concavity does not hold true even for very simple geometries, like $\Omega$ being a square (see [26, page 613]). On the other hand, the solution $u$ of (1.2) somehow gains the convexity of the domain: precisely $\sqrt{u}$ is concave, as proved by Makar-Limanov in [30] for dimension 2 and by Kennington in [23] for general $n$. Another classical result by Brascamp and Lieb [5] states that the first positive eigenfunction $u$ of the Laplace operator in a convex domain is log-concave, i.e. $\log (u)$ is concave. These examples suggest that in general one can look for some power concavity of the solution of the involved problem. We recall the following definition: a positive function $u$ is said $p$-concave for some $p \in(-\infty, \infty)$ if

$$
\left\{\begin{array}{l}
\frac{p}{|p|} u^{p} \text { is concave for } p \neq 0 \\
\log (u) \text { is concave for } p=0 .
\end{array}\right.
$$

Usually, 0-concave functions are said log-concave. When $p \rightarrow-\infty$ we get the notion of quasi-concavity: a function $u$ is said quasi-concave if all its super level sets are convex. Quasi-concavity is the weakest conceivable concavity property.

When dealing with a parabolic problem like (1.1), the geometry of the solution $u$ is influenced not only by the domain $\Omega$ but also by the initial datum $\varphi$. Moreover, the concavity of the solution can be intended in two different ways: spatial concavity, i.e. we can ask whether $u$ is (power) concave when regarded as a function of the spatial variable $x$ only at every fixed time, or spacetime concavity, i.e. (power) concavity with respect to $(x, t)$. Most of the results in literature regard spatial concavity. For instance, it is easily seen that, when $\Omega=\mathbf{R}^{n}$, every solution $u$ of (1.1) with moderate growth at space infinity preserves the spatial concavity of $\varphi$ at every time $t>0$ : just consider any direction $\xi$, then $u_{\xi \xi}$ solves the heat equation in $\mathbf{R}^{n}$ with initial datum $\varphi_{\xi \xi} \leq 0$, hence $u_{\xi \xi} \leq 0$ for every direction $\xi$ and $u$ is concave in $x$ for every time $t$. Related results and extensions can be found in [9], [19] and [27], for instance. It is also well known that heat flow pushes forward the spatial log-concavity of the initial datum in a convex domain, as proved by Brascamp and Lieb in the already quoted classical paper [5]. An alternative proof of this result can be obtained with the methods of another famous paper by Korevaar [26]. Further investigations on spatial log-concavity in parabolic problems can be found in [10]. Moreover, Lee and Vázquez proved in [28] that, if the initial function $\varphi$ has a compact support, then the solution of (1.1) is spatially log-concave after a finite time. Also Kennington considered the spatial power concavity of solutions of parabolic problems (see [23] and [24]). The quoted results show that log-concavity has a special role in the parabolic case; and indeed weaker properties than log-concavity are not necessarily preserved by the heat flow (see [14] and [15]).

On the other hand, space-time concavity properties are also very interesting (and may be more natural) for parabolic problems. Inspired by [4], the authors of this paper introduced in [16] and [17] the notions of parabolic and $\alpha$-parabolic quasi-concavity, and studied quasi-concavity properties involving the space and the time variables jointly for particular parabolic boundary value problems in 
a convex ring. Furthermore, they developed in [18] the notion of $\alpha$-parabolic $q$-concavity for nonnegative functions and study parabolic power concavity properties of solutions to parabolic boundary value problems in a convex cylinder with vanishing initial datum and a suitable source term. See also $[6,7,8,13,31]$ for further results related to space-time concavity of solutions of parabolic problems.

In this paper we investigate the parabolic log-concavity of the solutions of (1.1) when $\Omega=\mathbf{R}^{n}$. Moreover, in the case where $\Omega$ is a bounded convex domain, we apply the results of [18] to study the $\alpha$-parabolic power concavity for the quantity

$$
U(x, t):=\int_{0}^{t} u(x, s) d s,
$$

which could be regarded as the cumulative heat at the point $x$ at time $t$.

The rest of this paper is organized as follows. In Section 2 we introduce some notation and recall some known results on concavity properties. In Section 3 we state the main theorems of this paper, Theorems 3.1 and 3.2, which are concerned with parabolic log-concavity for the solution of (1.1) in the case $\Omega=\mathbf{R}^{n}$. In Section 4 we prove Theorems 3.1 and 3.2. In Section 5 we consider problem (1.1) in the case where $\Omega$ is a bounded convex domain, and study $\alpha$-parabolic $q$-concavity of $U$.

Acknowledgements. The authors warmly thank an unknown referee for many helpful suggestions and comments that helped a lot to improve the paper, and especially for pointing out a mistake in the original version of Theorem 3.1.

The first author has been partially supported by the Grant-in-Aid for Challenging Exploratory Research (No. 25610023), Japan Society for the Promotion of Science. The second author has been partially supported by GNAMPA (Gruppo Nazionale per l'Analisi Matematica, la Probabilità e le loro Applicazioni) of INdAM (Istituto Nazionale di Alta Matematica).

\section{Preliminaries}

We introduce some notation and recall some preliminary properties of the solutions of the heat equation and quasi-concave functions.

For any $x \in \mathbf{R}^{n}$ and $r>0$, we put $B(x, r)=\left\{y \in \mathbf{R}^{n}:|x-y|<r\right\}$. For any set $D \subset \mathbf{R}^{n}$, we denote by $\chi_{D}$ the characteristic function of $D$, that is, $\chi_{D}(x)=1$ for $x \in D$ and $\chi_{D}(x)=0$ for $x \notin D$. For any nonnegative function $\varphi \in C(\Omega) \cap$ $L^{\infty}\left(\mathbf{R}^{n}\right)$, there is a unique nonnegative solution of (1.1) in the case $\Omega=\mathbf{R}^{n}$, that is the following

$$
(4 \pi t)^{-n / 2} \int_{\mathbf{R}^{n}} e^{-|x-y|^{2} / 4 t} \varphi(y) d y, \quad(x, t) \in \mathbf{R}^{n} \times(0, \infty) .
$$


2.1. Power concave and parabolic power concave functions. For $a, b>0$, $\lambda \in(0,1)$ and $p \in[-\infty, \infty]$, we define

$$
M_{p}(a, b ; \lambda)= \begin{cases}{\left[(1-\lambda) a^{p}+\lambda b^{p}\right]^{1 / p}} & \text { if } p \notin\{-\infty, 0, \infty\}, \\ a^{1-\lambda} b^{\lambda} & \text { if } p=0, \\ \max \{a, b\} & \text { if } p=\infty, \\ \min \{a, b\} & \text { if } p=-\infty,\end{cases}
$$

which is the $p$-(weighted) mean of $a$ and $b$ with ratio $\lambda$. Furthermore, for $a, b \geq 0$, we define $M_{p}(a, b ; \lambda)$ as above if $p \geq 0$ and

$$
M_{p}(a, b ; \lambda)=0 \text { if } p<0 \text { and } a \cdot b=0 .
$$

Definition 2.1. Let $K$ be a convex set in $\mathbf{R}^{m}$ and $p \in[-\infty, \infty] . A$ nonnegative function $v$ defined in $K$ is said p-concave if

$$
v((1-\lambda) x+\lambda y) \geq M_{p}(v(x), v(y) ; \lambda)
$$

for all $x, y \in K$ and $\lambda \in(0,1)$. Moreover, $v \geq 0$ is said $p$-concave in $\mathbf{R}^{m}$ if it has a convex support $K$ and it is $p$-concave in $K$. In the cases $p=0$ and $p=-\infty, v$ is also said log-concave and quasi-concave, respectively.

Then the following holds.

\section{LEMMA 2.1 .}

(i) If $v$ is $p$-concave, then $v$ is $q$-concave for every $q \leq p$.

(ii) For any $-\infty$-concave function $v$, define

$$
\alpha(v):=\sup \{\beta \in \mathbf{R}: v \text { is } \beta \text {-concave }\} .
$$

Then $v$ is $\alpha(v)$-concave.

Proof. Properties (i) and (ii) are well-known (see [23] for instance); we just give a sketch of the proof for reader's convenience. Property (i) follows from Jensen's inequality, which implies that $M_{q}(a, b ; \lambda) \leq M_{p}(a, b ; \lambda)$ if $q \leq p$. Property (ii) follows from the continuity of $M_{p}(a, b ; \lambda)$ with respect to $p \in$ $[-\infty,+\infty]$.

Notice that (i) implies that quasi-concavity is the weakest concavity property one can imagine.

For more details on power concave functions, we address the reader to [5] and [23]. Here we just recall the following result from [5, Corollary 3.5].

Proposition 2.1. Let $\alpha \geq-1 / n$ and let $F(x, y) \geq 0$ be $\alpha$-concave in $\mathbf{R}^{m} \times \mathbf{R}^{n}$. Then the function

$$
G(x)=\int_{\mathbf{R}^{n}} F(x, y) d y
$$

is $\gamma$-concave in $\mathbf{R}^{m}$ with $\gamma=\alpha /(1+n \alpha)$. In particular, if $F$ is log-concave, so is $G$. 
Next we follow $[16,17,18]$ and give the definition of $\alpha$-parabolic $q$-concavity for nonnegative functions in a convex cylinder. See also [4].

Definition 2.2. Let $\Omega \subseteq \mathbf{R}^{n}$ be a convex set, $Q=\Omega \times[0, \infty)$ and $\alpha, p \in$ $[-\infty, \infty]$. A nonnegative function $v \in C(Q)$ is said $\alpha$-parabolically $p$-concave if

$$
v\left((1-\lambda) x_{1}+\lambda x_{2}, M_{\alpha}\left(t_{1}, t_{2} ; \lambda\right)\right) \geq M_{p}\left(v\left(x_{1}, t_{1}\right), v\left(x_{2}, t_{2}\right) ; \lambda\right)
$$

for all $\left(x_{1}, t_{1}\right),\left(x_{2}, t_{2}\right) \in Q$ and $\lambda \in(0,1)$.

For $p=0$ and $p=-\infty$ we speak again of $\alpha$-parabolic log-concavity and quasi-concavity. Roughly speaking, for any $\alpha \in \mathbf{R} \backslash\{0\}, v$ is $\alpha$-parabolically $p$-concave in $Q$ if

- $v$ is a constant function in $Q$ for $p=\infty$;

- $v\left(x, t^{1 / \alpha}\right)^{p}$ is concave in $Q$ for $p>0$;

- $\log v\left(x, t^{1 / \alpha}\right)$ is concave in $Q$ for $p=0$;

- $v\left(x, t^{1 / \alpha}\right)^{p}$ is convex in $Q$ for $p<0$;

- the superlevel sets $\left\{(x, t) \in Q: v\left(x, t^{1 / \alpha}\right)>\mu\right\}$ are convex for every $\mu \geq 0$ for $p=-\infty$.

Obviously, if $v$ is $\alpha$-parabolically $p$-concave for some $\alpha \in[-\infty,+\infty]$, then $v(\cdot, t)$ is spatially $p$-concave at any fixed time $t$.

Remark 2.1. Due to the homogeneity of the heat equation with respect to time and space, the case corresponding to $\alpha=1 / 2$ plays a special role and a $\frac{1}{2}$ parabolically $p$-concave function is simply said parabolically $p$-concave. Analogously, thanks to the properties of the heat kernel in $\mathbf{R}^{n}$, a special role is also played by the case corresponding to $p=0$, that is log-concavity.

\section{Solutions in $\mathbf{R}^{n}$}

In this section we consider problem (1.1) in the case $\Omega=\mathbf{R}^{n}$, and state our theorems about the parabolic concavity properties of the solution.

Theorem 3.1. Let $\Omega=\mathbf{R}^{n}$ and let $u$ be the nonnegative solution of (1.1). If the initial function $\varphi \in C\left(\mathbf{R}^{n}\right) \cap L^{\infty}\left(\mathbf{R}^{n}\right)$ is such that

$$
\phi(x, t)=\varphi\left(t^{-\beta} x\right) \text { is log-concave in } \mathbf{R}^{n} \times(0, \infty)
$$

for some $\beta>0$, then the function

$$
v_{\beta}(x, t):=u\left(t^{-\beta} x, t^{-2 \beta}\right)
$$

is log-concave in $\mathbf{R}^{n} \times(0, \infty)$. Equivalently, the function

$$
w_{\beta}(x, t)=t \log u\left(t^{\beta-1} x, t^{2 \beta}\right)
$$

is concave in $\mathbf{R}^{n} \times(0, \infty)$. 
Notice that the conclusion of the above theorem can be also equivalently expressed by saying that the function $u\left(t^{-1} x, t^{-2}\right)$ is $\frac{1}{\beta}$-parabolically log-concave, or by saying that the function $u\left(t^{-\beta / 2} x, t^{-\beta}\right)$ is parabolically log-concave, or by similar statement referred to the function $w_{\beta}$. In any case, it is apparent that Theorem 3.1 implies the spatial $\log$-concavity of $u$ at every fixed time $t>0$.

As a corollary of Theorem 3.1 with $\beta=1$, we obtain:

THeOREM 3.2. Let $\Omega=\mathbf{R}^{n}$ and let $u$ be the nonnegative solution of (1.1). If $\varphi$ is the characteristic function of a convex set, then the function

$$
w(x, t)=\sqrt{t} \log u(x, t)
$$

is parabolically concave in $\mathbf{R}^{n} \times(0, \infty)$.

The proofs of Theorems 3.1 and 3.2 are given in Section 4 .

Remark 3.1. As said in the Introduction, Lee and Vázquez proved that any solution of (1.1) becomes spatially log-concave after a possibly large time $T>0$ when the initial function $\varphi$ has a compact support. One can wonder whether a similar property hold for space-time log-concavity, i.e. whether it is possible to find conditions on the initial datum $\varphi$ such that the solution $u$ surely becomes somehow space-time log-concave after a possibly large time $T$. This is an interesting question and we conjecture the answer to be positive for any initial datum with compact support.

The same question naturally arises for a bounded convex domain $\Omega$. In this case, following the suggestion of an unknown referee, we conjecture that some kind of large-time parabolic log-concavity hold for the solution of (1.1) when the initial datum $\varphi$ satisfies $\int_{\Omega} \varphi u_{1} d x>0$, with $u_{1}$ the first positive Dirichlet eigenfunction of the Laplace operator in $\Omega$.

In the rest of this section we discuss assumption (3.3) in order to show that there is plenty of functions $\varphi$ satisfying it.

First notice that (3.3) implies that $\varphi$ be log-concave in $\mathbf{R}^{n}$ (just fix $t=1$ ), i.e. it is positive in a convex set $K$ (possibly $K=\mathbf{R}^{n}$ ), vanishing outside of $K$ and there exists a convex function $h$ (assuming the conventional value $+\infty$ outside of $K$ ) such that

$$
\varphi(x)=e^{-h(x)} .
$$

Then assumption (3.3) asks $h\left(t^{-\beta} x\right)$ to be convex with respect to $(x, t)$.

Let $n=1$. Then $K$ must be an interval $I$ in $\mathbf{R}$. Assume $h$ is not a constant function in $\mathbf{R}$ and that $h(x) \rightarrow \infty$ as $|x| \rightarrow \infty$. We can assume, without loss of generality, that $0 \in I$ and

$$
h(0)=\min _{x \in \mathbf{R}} h(x)
$$


which implies that $h$ is decreasing for $x<0$ and it is increasing for $x>0$. Set $f(x, t)=h\left(t^{-\beta} x\right)$. Then

$$
\begin{gathered}
f_{x}=t^{-\beta} h^{\prime}, \quad f_{t}=-\beta x t^{-\beta-1} h^{\prime}, \\
f_{x x}=t^{-2 \beta} h^{\prime \prime}, \quad f_{t t}=\beta(\beta+1) x t^{-\beta-2} h^{\prime}+\beta^{2} x^{2} t^{-2 \beta-2} h^{\prime \prime}, \\
f_{x t}=-\beta t^{-\beta-1} h^{\prime}-\beta x t^{-2 \beta-1} h^{\prime \prime},
\end{gathered}
$$

where $h^{\prime}$ and $h^{\prime \prime}$ are calculated at $t^{-\beta} x \in I$ (and they exist almost everywhere by convexity). These imply that

$$
\operatorname{det}\left(D^{2} f\right)=\beta^{2} t^{-2 \beta-2} h^{\prime}\left[\frac{1-\beta}{\beta} t^{-\beta} x h^{\prime \prime}-h^{\prime}\right] .
$$

Then $f$ is convex if and only if $h$ (which is convex) satisfies

$$
\begin{cases}\frac{1-\beta}{\beta} \xi h^{\prime \prime}(\xi)-h^{\prime}(\xi) \leq 0 & \text { for } \xi<0 \\ \frac{1-\beta}{\beta} \xi h^{\prime \prime}(\xi)-h^{\prime}(\xi) \geq 0 & \text { for } \xi>0\end{cases}
$$

where $\xi=t^{-\beta} x \in I$. Notice that, if $h^{\prime} /\left(\xi h^{\prime \prime}\right)$ is bounded away from zero, i.e. there exists $\varepsilon>0$ such that $\xi h^{\prime \prime} / h^{\prime}>\varepsilon$ for every $\xi \in I$ (for instance when $h(x)=|x|^{\gamma}$ for some $\left.\gamma>1\right)$, then (3.4) is certainly satisfied for sufficiently small $\beta>0$. Moreover, in the case $\beta=1$, (3.4) (jointly with the fact that $h$ is decreasing for $x<0$ and it is increasing for $x>0$ ) implies $h^{\prime}(\xi)=0$ for every $\xi \in I$. Then $h$ must be a positive constant function in the interval $I$ and $+\infty$ outside $I$, that is,

$$
\varphi(x)=c \chi_{I}
$$

for some $c>0$.

Similarly one can consider a convex function $h$ which is increasing in $\mathbf{R}$ (and tends to $+\infty$ as $x \rightarrow+\infty$ or assumes the conventional value $+\infty$ for large positive $x$ ) or which is decreasing in $\mathbf{R}$ (and tends to $+\infty$ as $x \rightarrow-\infty$ or assumes the conventional value $+\infty$ for large negative $x$ ).

Let $n \geq 2$ and let us consider the case where $h$ is a $\gamma$-homogeneous convex function with $\gamma \geq 0$, that is, $h$ is convex and

$$
h(t x)=t^{\gamma} h(x) \text { for every } t>0 .
$$

Then, thanks to [16, Lemma A.1],

$$
f(x, t)=h\left(t^{-\beta} x\right)=t^{-\gamma \beta} h(x)
$$

is convex if and only if $h(x)^{1 /(1+\gamma \beta)}$ is convex. 


\section{Proofs of Theorem 3.1 and Theorem 3.2}

Proof of Theorem 3.1. It follows from (2.1) that

$$
\begin{aligned}
v_{\beta}(\xi, s) & =\left(4 \pi s^{-2 \beta}\right)^{-n / 2} \int_{\mathbf{R}^{n}} \exp \left(-\frac{\left|s^{-\beta} \xi-y\right|^{2}}{4 s^{-2 \beta}}\right) \varphi(y) d y \\
& =(4 \pi)^{-n / 2} \int_{\mathbf{R}^{n}} \exp \left(-\frac{|\xi-z|^{2}}{4}\right) \varphi\left(s^{-\beta} z\right) d z .
\end{aligned}
$$

Since $\varphi\left(s^{-\beta} z\right)$ is log-concave with respect to $(z, s)$, the function

$$
K(s, \xi, z):=\exp \left(-\frac{|\xi-z|^{2}}{4}\right) \varphi\left(s^{-\beta} z\right)
$$

is log-concave with respect to $(s, \xi, z)$. Then we deduce from Proposition 2.1 that $v$ is log-concave with respect to $(\xi, s)$, and the proof of the first sentence of the theorem is complete.

Then the assertion regarding the function $w_{\beta}$ is a straightforward consequence of the following interesting algebraic lemma, applied to the function $-\log v_{\beta}$.

Lemma 4.1. Let $v$ be a function in $\mathbf{R}^{n} \times(0, \infty)$. Then $v$ is convex in $\mathbf{R}^{n} \times(0, \infty)$ if and only if the function

$$
w(\xi, s)=s v\left(s^{-1} \xi, s^{-1}\right),
$$

is convex with respect to $(\xi, s) \in \mathbf{R}^{n} \times(0, \infty)$.

Proof. Assume that $w$ is convex, i.e.

$$
\begin{aligned}
& \left((1-\mu) s_{0}+\mu s_{1}\right) v\left(\frac{(1-\mu) \xi_{0}+\mu \xi_{1}}{(1-\mu) s_{0}+\mu s_{1}},\left((1-\mu) s_{0}+\mu s_{1}\right)^{-1}\right) \\
& \quad \leq(1-\mu) s_{0} v\left(\frac{\xi_{0}}{s_{0}}, s_{0}^{-1}\right)+\mu s_{1} v\left(\frac{\xi_{1}}{s_{1}}, s_{1}^{-1}\right)
\end{aligned}
$$

for $\xi_{0}, \xi_{1} \in \mathbf{R}^{n}, s_{0}, s_{1}>0$ and $\mu \in(0,1)$. Set

$$
s_{i}=t_{i}^{-1}, \quad \xi_{i}=s_{i} x_{i}, \quad \lambda=\frac{\mu s_{1}}{(1-\mu) s_{0}+\mu s_{1}},
$$

for $i=0,1$. Since

$$
1-\lambda=\frac{(1-\mu) s_{0}}{(1-\mu) s_{0}+\mu s_{1}}
$$


and

$$
\begin{aligned}
(1-\lambda) t_{0}+\lambda t_{1} & =\frac{1-\mu}{(1-\mu) s_{0}+\mu s_{1}}+\frac{\mu}{(1-\mu) s_{0}+\mu s_{1}} \\
& =\left((1-\mu) s_{0}+\mu s_{1}\right)^{-1}
\end{aligned}
$$

(4.5) reads

$$
v\left((1-\lambda) \xi_{0}+\lambda x_{1},(1-\lambda) t_{0}+\lambda t_{1}\right) \leq(1-\lambda) v\left(x_{0}, t_{0}\right)+\lambda v\left(x_{1}, t_{0}\right),
$$

which says that $v$ is convex with respect to $(x, t) \in \mathbf{R}^{n} \times(0, \infty)$. Similarly we see that $w$ is convex if $v$ is convex. Thus Lemma 4.1 follows.

Proof of Theorem 3.2. Let $K$ be a convex set. By the second assertion of Theorem 3.1 (and the discussion about parabolic concavity following Definition 2.2), it suffices to prove that the function

$$
\phi(x, t)=\chi_{K}(x / t)
$$

is log-concave with respect to $(x, t) \in \mathbf{R}^{n} \times(0, \infty)$. Let $\lambda \in(0,1)$. Since $\phi(x, t)=$ $\chi_{t K}(x)$, we have

$$
\phi\left(x_{0}, t_{0}\right)^{1-\lambda} \phi\left(x_{1}, t_{1}\right)^{\lambda}= \begin{cases}1 & \text { if } x_{0} \in t_{0} K \text { and } x_{1} \in t_{1} K \\ 0 & \text { otherwise }\end{cases}
$$

for all $\left(x_{0}, t_{0}\right)$ and $\left(x_{1}, t_{1}\right) \in \mathbf{R}^{n} \times(0, \infty)$. On the other hand, if $x_{0} \in t_{0} K$ and $x_{1} \in t_{1} K$, then

$$
(1-\lambda) x_{0}+\lambda x_{1} \in\left((1-\lambda) t_{0}+\lambda t_{1}\right) K
$$

and we have

$$
\phi\left((1-\lambda) x_{0}+\lambda x_{1},(1-\lambda) t_{0}+\lambda t_{1}\right)=1
$$

This together with (4.6) implies that

$$
\phi\left((1-\lambda) x_{0}+\lambda x_{1},(1-\lambda) t_{0}+\lambda t_{1}\right) \geq \phi\left(x_{0}, t_{0}\right)^{1-\lambda} \phi\left(x_{1}, t_{1}\right)^{\lambda}
$$

for all $\left(x_{0}, t_{0}\right)$ and $\left(x_{1}, t_{1}\right) \in \mathbf{R}^{n} \times(0, \infty)$, which implies that $\phi$ is log-concave with respect to $(x, t) \in \mathbf{R}^{n} \times(0, \infty)$. Thus Theorem 3.2 follows.

\section{Solutions in a bounded convex domain}

Let $\Omega$ be a bounded convex domain in $\mathbf{R}^{n}$ and let $u$ be the solution of (1.1). In this section we discuss how the power concavity of the initial datum $\varphi$ influences the solution $u$.

We recall the following result on parabolic power concavity for the heat equation with a source term (see [18, Theorem 2]). 
Proposition 5.1. Let $\Omega$ be a bounded convex domain in $\mathbf{R}^{n}, \quad D:=$ $\Omega \times(0, \infty)$ and $f$ a nonnegative function in $\Omega$. Let $w \in C^{2,1}(D) \cap C(\bar{D})$ satisfy

$$
\begin{cases}\partial_{t} w=\Delta w+f & \text { in } D, \\ w=0 & \text { on } \partial D .\end{cases}
$$

Then the following holds:

(i) if $f$ is $q$-concave in $\Omega$ for some $q \geq 1$, then $w$ is $\alpha$-parabolically $p$-concave in $\bar{D}$ with $p=q /(1+2 q)$ and $\alpha \geq 1 / 2$;

(ii) if $f$ is a positive constant in $\Omega$, then $\sqrt{w\left(x, t^{1 / \alpha}\right)}$ is concave in $\bar{D}$ for any $\alpha \geq 1 / 2$.

Proposition 5.1 shows that the power concavity of a source term compels some concavity of the solution with respect to space and time jointly. In this section we apply Proposition 5.1 to problem (1.1), and prove the following theorem on problem (1.1), which establishes the parabolic power concavity of

$$
U(x, t)=\int_{0}^{t} u(x, s) d s .
$$

Roughly speaking, the function $U$ represents the cumulative heat in $x$ at time $t$.

THEOREM 5.1. Let $\Omega$ be a bounded convex domain in $\mathbf{R}^{n}$. Let $u$ be the solution of (1.1), where $\varphi \in C(\Omega) \cap L^{\infty}(\Omega)$ and $\varphi \geq 0$ in $\Omega$. Then the following holds for the function $U$ defined by (5.8):

(i) if $\varphi$ is $q$-concave in $\Omega$ for some $q \geq 1$, then $U$ is $\alpha$-parabolically $p$-concave in $\bar{D}$ with $p=q /(1+2 q)$ and $\alpha \geq 1 / 2$;

(ii) if $\varphi$ is a positive constant in $\Omega$, then $\sqrt{U\left(x, t^{1 / \alpha}\right)}$ is concave in $\bar{D}$ for any $\alpha \geq 1 / 2$.

Proof. Since $u$ is a solution of (1.1), we easily see that $U$ satisfies

$$
\begin{cases}U_{t}=\Delta U+\varphi(x) & \text { in } D \\ U=0 & \text { on } \partial D\end{cases}
$$

where $D=\Omega \times(0, \infty)$. Then Theorem 5.1 immediately follows from Proposition 5.1.

Remark 5.1. We remark that Theorem 5.1 contains as a particular case concavity properties of positive solutions of

$$
-\Delta U=\varphi \quad \text { in } \Omega, \quad U=0 \quad \text { on } \partial \Omega,
$$

which corresponds to (5.8) with $t=+\infty$. In particular, we notice that a possible alternative definition of the torsion function of a set $\Omega$ is the following:

$$
U(x)=\int_{0}^{\infty} u(x, t) d x
$$


where $u$ is the solution of $(1.1)$ with initial value $\varphi \equiv 1$, see for example [1]. Then Theorem 5.1 implies the Makar-Limanov's result and its generalization to $n \geq 2$.

\section{REFERENCES}

[1] M. van DEn Berg, Estimates for the torsion function and Sobolev constants, Potential Anal. 36 (2012), 607-616.

[2] B. Bian AND P. GuAn, A microscopic convexity principle for nonlinear partial differential equations, Invent. Math. 177 (2009), 307-335.

[ 3 ] M. BiAnChINI AND P. SAlani, Power concavity for solutions of nonlinear elliptic problems in convex domains, Geometric properties for parabolic and elliptic PDE's, Springer INdAM, Ser. 2, Springer, Milan, 2013, 35-48.

[4] C. Borell, A note on parabolic convexity and heat conduction, Ann. Inst. H. Poincaré Probab. Statist. 32 (1996), 387-393.

[ 5 ] H. J. Brascamp and E. H. Lieb, On extensions of the Brunn-Minkowski and Prékopa-Leindler theorems, including inequalities for $\log$ concave functions, and with an application to the diffusion equation, J. Functional Anal. 22 (1976), 366-389.

[6] C. Chen, On the microscopic spacetime convexity principle for fully nonlinear parabolic equations I: Spacetime convex solutions, arXiv:1403.5077v3.

[7] C. CHEN, On the microscopic spacetime convexity principle of fully nonlinear parabolic equations II: Spacetime quasiconcave solutions, arXiv:1403.5078v3.

[8] C. CHEN AND B. Hu, A microscopic convexity principle for spacetime convex solutions of fully nonlinear parabolic equations, Acta Mathematica Sinica, English Series 29 (2013), 651-674.

[9] Y. Giga, S. Goto, H. Ishi and M.-H. SAto, Comparison principle and convexity preserving properties for singular degenerate parabolic equations on unbounded domains, Indiana Univ. Math. J. 40 (1991), 443-470.

[10] A. Greco AND B. Kawohl, Log-concavity in some parabolic problems, Electron. J. Differential Equations 1999 (1999), 1-12.

[11] P. Guan And L. XU, Convexity estimates for level sets of quasiconcave solutions to fully nonlinear elliptic equations, J. Reine Angew. Math. 680 (2013), 41-67.

[12] F. Hamel, N. Nadirashvili and Y. Sire, Convexity of level sets for elliptic problems in convex domains or convex rings: two counterexamples, arXiv:1304.3355v2.

[13] B. Hu AND X. MA, A constant rank theorem for spacetime convex solutions of heat equation, Manuscripta Math. 138 (2012), 89-118.

[14] K. Ishige AND P. Salani, Is quasi-concavity preserved by heat flow?, Archiv der Mathematik 90 (2008), 450-460.

[15] K. Ishige AND P. Salani, Convexity breaking of the free boundary for porous medium equations, Interfaces and Free Boundaries 12 (2010), 75-84.

[16] K. Ishige AND P. SAlani, Parabolic quasi-concavity for solutions to parabolic problems in convex rings, Math. Nachr. 283 (2010), 1526-1548.

[17] K. Ishige and P. SAlani, On a new kind of convexity for solutions of parabolic problems, Discrete Contin. Dyn. Syst., Ser. S. 4 (2011), 851-864.

[18] K. Ishige And P. SAlani, Parabolic power concavity and parabolic boundary value problems, Math. Ann. 358 (2014), 1091-1117.

[19] S. JAnson AND J. Tysk, Preservation of convexity of solutions to parabolic equations, J. Differential Equations 206 (2004), 182-226. 
[20] P. JuUtinen, Convexity of solutions to boundary blow-up problems, Commun. Pure Appl. Anal. 12 (2013), 2267-2275.

[21] B. KAwoHL, Rearrangements and convexity of level sets in P.D.E., Lecture notes in mathematics 1150, Springer, Berlin, 1985.

[22] B. KawoHL, Qualitative properties of solutions to semilinear heat equations, Exposition. Math. 4 (1986), 257-270.

[23] A. U. Kennington, Power concavity and boundary value problems, Indiana Univ. Math. J. 34 (1985), 687-704.

[24] A. U. Kennington, Convexity of level curves for an initial value problem, J. Math. Anal. Appl. 133 (1988), 324-330.

[25] S. KIM AND K.-A. LEE, Asymptotic behavior in degenerate parabolic fully nonlinear equations and its application to elliptic eigenvalue problems, J. Differential Equations 254 (2013), 3259-3306.

[26] N. J. KorevaAR, Convex solutions to nonlinear elliptic and parabolic boundary value problems, Indiana Univ. Math. J. 32 (1983), 603-614.

[27] P.-L. Lions And M. Musiela, Convexity of solutions of parabolic equations, C. R. Math. Acad. Sci. Paris 342 (2006), 915-921.

[28] K.-A. LeE AND J. L. VÁzqUEZ, Geometrical properties of solutions of the porous medium equation for large times, Indiana Univ. Math. J. 52 (2003), 991-1016.

[29] X. MA, S. SHI AND Y. YE, The convexity estimates for the solutions of two elliptic equations, Comm. Partial Differential Equations 37 (2012), 2116-2137.

[30] L. Makar-Limanov, The solution of the Dirichlet problem for the equation $\Delta u=-1$ in a convex region, Mat. Zametki 9 (1971), 89-92.

[31] G. Porru and S. Serra, Maximum principles for parabolic equations, J. Aust. Math. Soc. 56 (1994), 41-52.

\author{
Kazuhiro Ishige \\ Mathematical InSTITUTE \\ TOHOKU UNIVERSITY \\ Aoba, Sendai 980-8578 \\ JAPAN \\ E-mail: ishige@math.tohoku.ac.jp \\ Paolo Salani \\ Dipartimento di Matematica 'U. Dini' \\ Viale Morgagni 67/A \\ 50137 FIRENZE \\ ITALY \\ E-mail: paolo.salani@unifi.it
}

